\title{
Incorporating Uncertainty into Reliability Constraints
}

General reliability issues are large relative to total electricity sales and large blackouts are disruptive to society. These costs are hidden from the system and distributed throughout the economy. New dispatch models are needed to account for these probabilistic risks and incorporate uncertainty into their operation. At the intersection of economic efficiency and reliability are dynamic line limits and transmission congestion, where these issues are explored.

\section{Eric Anderson}

Eric Anderson is a graduate student at the University of Wisconsin, Madison, pursuing his Ph.D. in Industrial Engineering. He received an M.S. in Industrial Engineering in 2011, and a B.S. in Applied Math, Engineering, and Physics in 2009. His primary research is on computational models for risk and reliability of bulk power systems. Using tools from the optimization and operations research fields, he looks to quantify and price risk in a day-to-day setting with dispatch models, as well as making the power grid more robust to large-scale cascading power failures.

This work was made possible through Research Assistant funding at University of Wisconsin Madison by the author's advisor Jeff Linderoth through grants with the Department of Energy. Thanks are given for discussions of related work with Jeff Linderoth and Jim Luedtke, which were extremely valuable.

Bulk power systems rely on dispatch models to clear the economic markets for power supply in a timely manner. These models are critical to ensure that the market is cleared while minimizing cost and maintaining a set of reliability constraints. In its most common form, the DC power flow model is used to clear markets for both real-time and day-ahead operation, where the day-ahead operation has the additional complexity of committing slow-ramping resources. One set of reliability constraints is line thresholds that the system must maintain continuously as well as the system's resiliency to $\mathrm{N}-1$ contingencies. The hard line limits are developed from thermal characteristics of lines, a set of environmental conditions, and assumptions on the acceptable risk level of failure. 
To illustrate the dynamics between these reliability constraints and uncertainty, we will take a deep dive into transmission congestion and its intersection with reliability. To understand the connection with transmission congestion, we start with general reliability issues where we see momentary power outages constitute two-thirds of the cost and the commercial sector is hit the hardest. Large blackouts are often the result of extreme weather events or cascading power failures. We then review the Northeast blackout of 2003, where lines failed below their nominal capacity and contributed to system instability. Here we investigate the dynamic equilibrium in this complex system where blackouts of all sizes occur. Cascading power failures are linked with a high utilization of the bulk transmission system. Since these rare events are disproportionately costly to society, we look at understanding this risk and appropriately constraining the system to reduce the likelihood of occurrence.

Several models now account for uncertainty in load and wind generation and are able to enforce these reliability constraints probabilistically. Additionally, there are models that look at risk from a system's perspective and enforce a system risk constraint. This perspective is most closely linked with the critical point theory in cascading power failure research and may prove useful to reduce the likelihood of large blackouts. With the abundance of data in power systems and the increased capabilities of the smart grid, models need to be developed to incorporate the many sources of uncertainty and enforce system reliability constraints in a probabilistic manner.

\section{Reliability in Power Systems}

The electricity grid is held to a higher reliability standard than other services since it is a critical infrastructure for society. Even though the power system maintains a very high level of reliability, power interruptions still have large costs to consumers. After a discussion of the economic costs, we will examine trends in power outages for the North American grid.

The economic loss caused by power interruptions to electricity consumers in the United States for 2001 was estimated at $\$ 79$ billion (LaCommare \& Eto., 2006). This can be compared to the total revenue of electrical sales in the year 2001 of $\$ 247$ billion (U.S. Energy Information Agency, 2001). The single event of the Northeast blackout in 2003 was estimated to have a total impact on U.S. workers, consumers, and taxpayers as a loss of approximately $\$ 6.4$ billion (Anderson \& Geckil, 2003). This cost is hidden from the system and distributed through the economy. 
Power outages can be categorized based upon the time of lost service. The estimate of $\$ 79$ billion was found through a bottom-up study by classifying and quantifying outages and looking at the average cost of outages in each category. Momentary interruptions last less than five minutes but account for two-thirds of the cost and sustained interruptions, which are anything greater than five minutes and account for one-third of the cost. The commercial sector bears a large portion of these costs, around 72 percent; industrial accounts for around 26 percent, and residential is around 2 percent (LaCommare \& Eto., 2006).

A number of studies have been conducted on this issue since the Northeast blackout of 2003 brought reliability issues to the forefront. Hines et al. provides a nice walkthrough of the two largest outage datasets from DOE and FERC. It finds that all of the top 15 blackouts from 1984 to 2006 were due to extreme weather events and cascading power failures. They looked at the five following hypotheses and used the data set to either reject or accept the premise (Hines, Apt, \& Talukday, 2009):

- Blackout frequency has decreased with time (reject);

- Blackout frequency does not change seasonally (reject);

- Blackout probability does not change with time of day (reject);

- Blackout sizes follow a power-law probability distribution (support), and

- Blackout sizes and restoration times are positively correlated (reject).

A recent grid resilience report adds further to this breakdown and shows that 80 percent of power failures are due to weather events, which cost between $\$ 30$ and $\$ 80$ billion a year (President's Council of Economic Advisers; U.S. Department of Energy's Office of Electricity Delivery and Energy Reliability; White House Office of Science and Technology, 2013). These indirect costs imposed on society are related to the loss of electric power for consumers. This differs from the direct cost related to reliability constraints when dispatch models are solved and certain reliability constraints are tight. Appropriate reliability constraints that increase dispatch cost should ideally reduce indirect costs due to outages and power quality issues. Direct costs are measurable, certain, and, depending on the amounts, preferable to electric outages and quality issues.

Some emerging conditions on the grid may make protection more important and more difficult. The bulk power system is more variable due to the introduction of deregulated wholesale markets. As the transmission infrastructure has not received large investments over the last three to four decades, the utilization of the transmission infrastructure has increased. The 
increased penetration of renewable energy production also increases stress on the power system. Wind and solar are both variable-generation sources that do not actively control the amount of electricity being produced. The system needs to maintain ample ramping capability in order to maintain stability. These stresses can be reduced by improving the quality of forecasting efforts on various time scales. In addition, these technologies are geographically constrained by their fuel source availability, winds and solar irradiation. However, these natural fuel sources do not align with large demand centers, so the transmission system is needed to efficiently distribute the energy produced.

Electric vehicles adopted in masse have a potential to offer useful services to the power grid through the use of a sizeable amount of energy storage in aggregate. However, they also represent a considerable stress on the system in different spots and ways. The ideal car battery system has a quick charge, that is, an ability to transfer the energy from the grid to the battery extremely quickly. This allows consumers to use charging stations similarly to the current gasoline stations for combustion engines. A charging station capable of charging many cars has extremely high and unpredictable volatility that the system needs to protect against. Without real-time prices getting to these loads, these will continue to be added stresses to the system (Anderson E. , 2014).

\section{Northeast 2003 Blackout}

The Northeast blackout of 2003 began in the Cleveland-Akron, Ohio, area of the Eastern Interconnection. This area had a history of problems of low voltages due to the relatively high amount of imported power. In 1998, the system was becoming unstable and everything other than load shedding was done to fix the problem. It was shown to not be within reliability standards, and regulations were loosened instead of addressing the problem. A transformer problem led American Electric Power (AEP), a control area entity, to perform a reliability study on the neighboring control area operated by First Energy (FE). The study again showed voltage instability problems.

The summer of 2003 was fairly typical with less than historical peak load. While the load was less than historic peak, it was consistently greater than the forecasted load. High temperatures, creating a greater air conditioning load, contributed to reactive power shortages. The voltages were low in Cleveland-Akron during the week, but within the new operating limits (92 percent to 105 percent). A group of shunt capacitors, out of service for planned maintenance, 
further reduced reactive power supply. Also, a large nuclear reactor, Davis-Besse, was in a longterm forced-outage state. This plant is normally able to provide a large amount of real power as well as reactive power support.

A total of three lines tripped at below operating capacity due to tree contact, starting with Harding-Chamberlin (44 percent of capacity) at 15:05. Then the Hanna-Juniper line tripped (88 percent normal and emergency rating) at 15:32. The Star-South Canton line had multiple tree contacts (55 percent) starting at 14:27, and finally tripped off for good (93 percent emergency rating) at 15:41. There were no prior sustained outages from tree contact in the previous years and FE used vegetation management practices consistent with the industry. When designing line limits, the thermal ratings are based on many variables including ambient temperatures and wind speeds. A combination of higher-than-nominal ambient temperature and lower wind speeds reduced the cooling capacity of lines.

In the United States, 45 million people lost power totaling 61,800 MW in Ohio, Michigan, Pennsylvania, New York, Vermont, Massachusetts, Connecticut, and New Jersey. The U.S. loss estimate was between $\$ 4$ billion and $\$ 10$ billion. Another 10 million people from Canada lost power, leading to an estimated 18.9 million lost man-hours and \$2.3 billion in lost manufacturing shipments. There were at least 11 fatalities, power took up to four days to return, and rolling blackouts continued in Ontario for the following week. The formation of a large island, based off of hydro plants in western New York and Canada, was the basis for system restoration (U.S.-Canada Power System Outage Task Force, 2004).

\section{Dynamic Equilibrium}

The current power system has evolved throughout the last century due to economic and reliability issues and the responses of the operating entities to these forces. The power system has self-organized into a dynamic equilibrium where blackouts of all sizes occur. The average frequency of blackouts in the United States is 13 days and has been the same for 30 years, which represents this equilibrium (Dobson I. , Carreras, Lynch, \& Newman, 2001).

The opposition of the slow time scale force of growth in load and system capacity and fast time scale of cascading power failures and outages produced a dynamic equilibrium that can be seen in real world data. The blackout size distribution follows a power-law distribution making large, costly blackouts more likely. On a slow time scale, there are several things that happen to the electricity grid. The first main force is the slow growth in load with around 0.7 
percent growth per year for first decade of 21st century (U.S. Energy Information Agency, 2014). This has the effect of reducing the available capacity margins on power lines and increasing the likelihood of failures as well as possibly further constraining economic dispatch. While the slow time scale is progressing, random exogenous events, acts of nature, happen to outage individual components. Individual blackouts triggered by random events (equipment failure, weather, vandalism, attack) can become widespread through a series of cascades. The engineering response to blackouts in operating policies, maintenance, equipment and controls has the effect of increasing margins on the slow time scale. These forces push against each other and settle into an equilibrium (Carreras, Lynch, Dobson, \& Newman, 2004).

These opposing forces find an equilibrium. The equilibrium tends to be near critical points, which are points that have maximum power flow through the network for the nominal system capacity. The system self-organizes toward these points, which maximize efficiency of its assets. When the system approaches these critical points, the power flow becomes limited due to two possible causes:

- The power flows are limited due to transmission line constraints. This type of critical point has larger blackouts, but happen less frequently. In addition, the blackouts typically have multiple lines tripping.

- The power flows are limited due to generation capacity. This results in frequent blackouts, but of smaller size.

However, it is also possible to be in an operating regime that is close to both types of critical points simultaneously. When this is the case, blackouts of all sizes occur (Dobson I. , Carreras, Lynch, \& Newman, 2007). While this operating regime may not be good from a reliability standpoint, it has the desirable characteristic of being able to deliver the maximum power for the system. That is, when these two points are balanced, the system is capable of maximum throughput from its generators to its loads with minimal excess capacity. This is important from an economic perspective and a critical reason the system self-organizes to these types of points. This type of point tends to be the cheapest way to supply all the loads with power, while satisfying the minimum system reliability standards.

\section{Deterministic versus Probabilistic Approach}

From the Northeast blackout recount, we note that even staying below conservative hard line limits, there is still a risk of line failure. The probabilistic risks should not be hidden from the system, but instead incorporated into models so that a tradeoff between risk and cost can be 
made. These deterministic, hard-threshold, reliability constraints are inadequate as our grid moves forward on multiple fronts. First, we have access to, and a better understanding of, the current state of the system. This is accomplished through increased deployment of sophisticated sensors capable of determining power flows, line outages, line sag, and much more.

Additionally, the increased data output of the system allows for a more complete forecasting of future loads and generation. While demand has always been forecasted, increased granularity and its interaction with weather dependent variables allows a better characterization of the uncertainty of the system. While only the mean is used currently in the deterministic OPF framework, we have a very good understanding of the distribution of load over short time frames.

The heavy emphasis on smart grid technologies allows us to better characterize the uncertainty in the system. These technologies give us better knowledge of system status and better understanding of assumptions of uncertainty. We need models to take advantage of this. A lot of work has been put into forecasting wind in short to mid time frames (six hours and less). As the time frame shortens, the uncertainty in wind forecasts drastically decreases. Additionally, the uncertainty in wind is an order of magnitude higher than the uncertainty in demand so the ability to characterize this is important for high penetration of wind generation (Jeon, Lamadrid, Mo, \& Mount, 2015). Ignoring this well characterized uncertainty in dispatch models lets the value of optimal recourse escape our system.

The uncertainty faced by the system is inherent and will never be closed through increased sensing and forecasting technologies. Additionally, the flexibility provided by demand response, energy storage, and electric vehicles provides mechanisms for recourse to counter this inherent uncertainty. While these technologies are receiving large research and development efforts, they are mature enough for deployment. A significant barrier to entry for these technologies is the business models and the system operating models to account for, price, and value these technologies according to the costs and benefits to the system. Once we acknowledge this inherent uncertainty in the system, we can begin to appropriately price stresses on the grid and ensure system constraints are value adding.

Over the course of the five-minute interval dispatch period, demand fluctuates from its expectation. This fluctuation can be due to the behaviors of aggregated residential load, commercial or industrial processes, or even power production for renewable sources such as 
wind and solar. Currently, ancillary services such as reserves and regulation support are acquired to deal with this uncertainty. This uncertainty is beginning to be handled for the traditional line threshold model of OPF using chance constraints. These chance constraints enforce the standard line threshold models probabilistic to ensure that the constraints are violated a small fraction of the time. Several extensions are made which involve arbitrary slack distribution (Bienstock, Chertkov, \& Harnett, 2012) and applications to large penetration of wind farms with HVDC (Vrakopoulou, et al., 2013).

\section{A. Transmission congestion, dynamic line limits and reliability}

Dynamic line limits are a low-hanging fruit for smart grid implementation due to their relatively low capital cost and ability to increase asset utilization. Dynamic line limits take advantage of a finer level of data input both geographically and temporally to calculate transmission element's capacity in a near-real-time system. A transmission line is typically constrained due to a fixed clearance requirement and the amount of sag induced by a given current flow. The amount of sag in a conductor is heavily influenced by environmental conditions, primarily wind speed and direction, solar radiation, and the ambient temperature. In the static line limit world, the year is typically divided coarsely into seasons and the environmental conditions are characterized by the worst-case scenario. This ensures that the transmission element is drastically underutilized, since worst-case scenarios are infrequent. Additionally, the system has no ability to adapt to a scenario that is worse than the input assumptions used. With the addition of sensing equipment and algorithms to determine the given sag for the current environmental conditions, the system is able to increase utilization of the transmission system, relaxing constraints and increasing economic efficiency, and the ability to recognize and increase reliability in worst case scenarios.

There is a hard limit on transmission lines that causes them to trip with certainty (due to protective relay elements); however, the system is operated far away from these points at or below their nominal capacity. If the system were operating close to these points, random fluctuation in power injects would cause the system to trip much more regularly than is seen. The nominal capacity of transmission elements are based on thermal characteristics of the line as well as a set of environmental characteristics that represent the worst-case scenario for different operating times. Typically, there may be a summer rating and a winter rating for each transmission element, where the winter rating has colder ambient temperature, thus less heating 
and less sagging. Dynamic line limits are being explored to account for current environmental conditions (Bucher, Vrakopoulou, \& Andersson, 2013) (Yip, An, Lloyd, Aten, \& Ferri, 2009) (Wang, Sheng, \& Jiang, 2011) (Zhang, Pu, McCalley, Stern, \& Jr. Gallus, 2002).

While this system increases the granularity for system constraints, it still is a deterministic system that ignores the inherent uncertainty. First, the generations and loads are uncertain, but at least can be well characterized. Wind generation's deviation from good forecasts has been shown to be normally distributed. This implies that the branch flows for the system will also be normally distributed, since for small deviations over short time intervals, a linear assumption can be made for branch flow sensitivities. In addition to operators not knowing the branch flow exactly, they must estimate environmental conditions as well. Both these factors combine so that the actual sag on the line is uncertain. The risk from line sag is due to the potential of a fault to earth. Thus, the risk of a fault is also dependent upon vegetation growth and trimming practices, adding additional uncertainties and human components to the risk (U.S.Canada Power System Outage Task Force, 2004) (Energy Sector Planning and Analysis, 2014). Finally, the line thresholds are determined based upon a predetermined risk level for faults under the assumption that the line is continuously loaded at the rated current (Seppa, 2007). It is at this last step that the probabilistic information is converted into a deterministic equivalent.

This conversion to a deterministic constraint removes important information for system reliability by using an extremely coarse representation and removes flexibility from the system to change its desired risk level. First, let's understand how this conversion crudely approximates the risk the constraint was designed to enforce. With the fixed line threshold determined based off the predefined risk preference, when solving the optimization problem it does not differentiate between 80 percent and 100 percent loading since both are feasible. However, once the flow crosses that threshold, say 100.1 percent, the system is infeasible and is considered at infinite risk. This implies the risk characteristics of a lightly loaded system and a maximally loaded system are equal. However we know this not to be true. Large blackouts, due to cascading power failures, occur when the transmission system is highly utilized. Additionally, blackouts are seasonally dependent and happen on shoulder or near-peak loading scenarios (Newman, Carreras, Degala, \& Dobson, 2011).

A reasonable assumption is that as the loading increases the risk of line failure also increases. Instead of hiding this line failure risk behind a hard threshold capacity constraint, this 
risk should be acknowledged and used to help in dispatch procedures and visualization and system understanding. Once the risk is brought to the forefront, the calculated value can be compared to empirical data and techniques refined to characterize this line failure risk.

\section{B. System perspective}

We are now seeing reliability constraints that focus on the system perspective. For large blackouts, individual line outages are only part of the picture. In the cascading failure research, we saw that large blackouts are correlated with the critical point where the system is constrained by the transmission infrastructure. This is not the case where one line is at capacity and it is otherwise lightly loaded. This critical point is characterized by a large subset of lines at or near their capacity. A risk-based security constrained optimal power flow model is now incorporating constraints of this type. A risk severity is developed to incorporate the individual line risks into a system risk measure. This system risk is then constrained and a tradeoff is developed between reliability and cost. Having an easily controllable trade-off allows the dispatcher to take into account current system information and risk tolerance (Wang, Zhang, McCalley, Zheng, \& Litvinov, 2014).

\section{Conclusion}

We began by exploring general reliability issues on the bulk power system. A large portion of these costs is classified as momentary power outages, less than five minutes, and the commercial sector is most affected. We then look at the Northeast blackout of 2003, which is in the class of large blackouts. These disruptive and costly events are primarily due to weather events and cascading power failures. A connection between these reliability issues and cascading power failures is made. These cascading power failures are more likely when the transmission system is heavily loaded.

In order to reduce these indirect costs distributed through the economy, we want to incorporate the probabilistic risk of these events into the dispatch models. One primary component of this risk is the inherent uncertainty in the system due to wind generation and aggregate load variation, which has the effect of making branch flows uncertain. The other

primary component is the transformation of probabilistic risk information into deterministic hard threshold constraints. This transformation hides the inherent risk from operators, provides a course representation of this risk, and doesn't allow for a tradeoff between risk and cost. The technologies of the smart grid will be well equipped to respond to these stresses, but there must 
be operational and business models to point them in the right direction. Dynamic line limits is one of the low-hanging fruits of the smart grid and is at the intersection of economic efficiency and reliability of the bulk power system.

Power systems' reliability constraints need to transition to a systems perspective that recognizes the inherent uncertainty in the system. Constraints should be value-adding and done in probabilistic terms to not give the illusion of a perfectly secure system while operating within those bounds. These models need to integrate the many sources of uncertainty from weather, generation, and load with the trade-off between economic efficiency and reliability. This can be seen most clearly within the discussion of hard thermal line limits and the modern dynamic line limits incorporating real-time access to weather and line sag conditions. These models need to go further. They also need to account for the uncertainty in branch flows like the recent chance constraint models seen in literature. Additionally, instead of individual line constraints to ensure reliability, the constraints should be seen from a systems perspective so that highly utilized transmission systems are transparently risky in the dispatch models. By tracking and constraining these probabilistic risk measures, operators can have a proper discussion of the tradeoff between economic efficiency and system reliability.

\section{References}

Anderson, E. (2014). Real-time pricing for charging electric vehicles. The Electricity Journal, 105-111.

Anderson, P. L., \& Geckil, I. K. (2003). Northeast Blackout Likely to Reduce U.S. Earnings by \$6.4. Anderson Economic Group.

Bienstock, D., Chertkov, M., \& Harnett, S. (2012). Chance Constrained Optimal Power Flow: Risk-Aware Network Control under Uncertainty. ArXiv e-prints, September.

Bucher, M., Vrakopoulou, M., \& Andersson, G. (2013). Probabilistic n-1 security assessment incorporating dynamic line ratings. IEEE Power and Energy Society General Meeting.

Carreras, B. A., Lynch, V., Dobson, I., \& Newman, D. E. (2004). Complex dynamics of blackouts in power transmission systems. Chaos, 14(3):643-652.

Dobson, I., Carreras, B. A., Lynch, V. E., \& Newman, D. E. (2001). An initial model for complex dynamics in electric power system blackouts. 34th Hawaii International Conference on System Science, (pp. 705-709).

Dobson, I., Carreras, B. A., Lynch, V. E., \& Newman, D. E. (2007). Complex systems analysis of series of blackouts: cascading failure, critical points, and self-organization. Chaos: An Interdisciplinary Journal of Nonlinear Science, 17(2).

Energy Sector Planning and Analysis. (2014). Dynamic line rating system. Smart Grid Demonstration Program. 
Hines, P., Apt, J., \& Talukday, S. (2009). Large blackouts in North America: Historical trends and policy implications. Energy Policy, 37:5249-5259.

Jeon, W., Lamadrid, A. J., Mo, J. Y., \& Mount, T. D. (2015). The controllability of real things: planning for wind integration. The Electricity Journal, 19-28.

LaCommare, K. H., \& Eto., J. H. (2006). Cost of power interruptions to electricity consumers. Energy, 31:1845-1855.

Newman, D., Carreras, B., Degala, N., \& Dobson, I. (2011). Risk metrics for dynamic complex infrastructure systems such as the power transmission grid. Maui, HI, 2082-2090.

President's Council of Economic Advisers; U.S. Department of Energy's Office of Electricity Delivery and Energy Reliability; White House Office of Science and Technology. (2013). Economic benefits of increasing electric grid resilience to weather outages. Executive Office of the President.

Seppa, T. O. (2007). Reliability and real time transmission line ratings. The Valley Group - A Nexans Company.

U.S. Energy Information Agency. (2001). Monthly Electric Utility Sales and Revenue Report. Retrieved 11 30, 2014, from http://www.eia.gov/electricity/data/eia826/

U.S. Energy Information Agency. (2014, 10 30). Energy Information Agency. Retrieved from http://www.eia.gov/electricity/data/eia826/

U.S.-Canada Power System Outage Task Force. (2004). Final Report on the August 14, 2003 Blackout in the United States and Canada: Causes and Recommendations. U.S. Department of Energy; Natural Resources Canada.

Vrakopoulou, M., Chatzivasileiadis, S., Iggland, E., Imhof, M., Krause, T., Makela, O., . . . Andersson, G. (2013). A unified analysis of security-constrained opf formulations considering uncertainty, risk, and controllability in single and multi-area systems. Bulk Power System Dynamics and Control.

Wang, K., Sheng, G., \& Jiang, X. (2011). Risk assessment of transmission dynamic line rating based on monte carlo. Power Engineering and Automation Conference, 2:398-402.

Wang, Q., Zhang, G., McCalley, J., Zheng, T., \& Litvinov, E. (2014). Risk-based locational marginal pricing and congestion management. IEEE Transactions on Power Systems, PP(99):1-11.

Yip, T., An, C., Lloyd, G., Aten, M., \& Ferri, B. (2009). Dynamic line rating protection for wind farm connections. Integration of Wide-Scale Renewable Resources Into the Power Delivery System.

Zhang, J., Pu, J., McCalley, J., Stern, H., \& Jr. Gallus, W. (2002). A bayesian approach for shortterm transmission line thermal overload risk assessment. IEEE Transactions on Power Delivery, 17(3):770-778. 